\title{
Recovering Inner Slices of Translucent Objects by Multi-frequency Illumination
}

\author{
Kenichiro Tanaka ${ }^{1,2}$ \\ Yasuhiro Mukaigawa ${ }^{2}$ \\ Hiroyuki Kubo ${ }^{2}$ \\ Yasuyuki Matsushita ${ }^{1,3} \quad$ Yasushi Yagi $^{1}$ \\ ${ }^{1}$ Osaka University $\quad{ }^{2}$ Nara Institute of Science and Technology ${ }^{3}$ Microsoft Research Asia \\ http://www.am.sanken.osaka-u.ac.jp/ tanaka/projects/multi-freq.html
}

\begin{abstract}
This paper describes a method for recovering appearance of inner slices of translucent objects. The outer appearance of translucent objects is a summation of the appearance of slices at all depths, where each slice is blurred by depth-dependent point spread functions (PSFs). By exploiting the difference of low-pass characteristics of depthdependent PSFs, we develop a multi-frequency illumination method for obtaining the appearance of individual inner slices using a coaxial projector-camera setup. Specifically, by measuring the target object with varying the spatial frequency of checker patterns emitted from a projector, our method recovers inner slices via a simple linear solution method. We quantitatively evaluate accuracy of the proposed method by simulations and show qualitative recovery results using real-world scenes.
\end{abstract}

\section{Introduction}

Translucent objects have complex appearance. It is a superposition of light rays emitted from inner slices at every depths, blurred by subsurface scattering. Because seeing internal appearance of objects is of broad interest in medical and art analyses and industry inspection, various imaging techniques have been developed in the past. In particular, since the translucency effect becomes significant for many materials in near infrared (NIR) wavelengths, infrared photography is used as one of common techniques for achieving this goal. For example, it is used for observing inner layers of oil paintings that tell us the drawing technique, growth, history, and/or authenticity of old age painters.

One of the major challenges in observing inner layers of translucent objects is to separate inner appearances with properly dealing with scattering. To overcome this problem, we develop a multi-frequency illumination method, which can recover sharp appearance of inner slices at a desired depth with explicitly removing scattering blur. Compared with conventional techniques that aim at a similar goal, our method is faster and safer than the X-ray fluorescence tech- nique [5], and sharper results can be obtained differently from infrared reflectography [9].

Our method exploits the fact that the spread of light due to scattering has dependency on the depth of inner layer where light rays are emitted. By modeling the light spreads as depth-dependent point spread functions (PSFs) and utilizing their depth-dependent low-pass characteristics, we develop a method for recovering inner layer appearances from a set of images taken under a variant of high-frequency illumination [26]. Specifically, our method uses a spatial pattern projection with varying the pattern pitch - we call this multi-frequency illumination. Our multifrequency illumination method allows us to separate direct (high-frequency) and global (low-frequency) components as in [26], yet at various frequency levels that define highand low-frequencies. Our method uses the direct component observations for recovering the appearance of inner slices, which are related to the direct components via depthdependent PSFs.

The key contributions of this paper are twofold. First, we describe the relationship between depth inside a translucent object and its PSF by a physically motivated scattering model. Second, using the relationship, we develop a method for recovering the appearance of inner slices using varying pitch pattern projection. We implement a coaxial projectorcamera setup for measuring real-world objects, and show the recovery results using oil painting, mural, and paper scenes.

\section{Related work}

As our work aims to recover slices from superposed observation, layer decomposition techniques $[29,27,19]$ from super-imposed images are related. These methods are designed for scenes with semi-transparent layers that do not exhibit multiple scattering; thus, the image formation model is represented by simple alpha blending. Our method is designed for translucent scenes where significant multiple scattering is observed.

Our work is related to descattering methods in computational photography in that our goal is to recover sharp slices 
inside a scattering medium. Narasimhan et al. [25]'s and Gu et al. [10]'s methods sharpen images of a target scene in muddy liquid by precisely modeling single scattering. Their methods work well for those scenes that do not exhibit multiple scattering. Fuchs et al. [8] use multiple cameras and projectors for descattering using a confocal imaging technique for the purpose of recording a solid target in scattering media. Duran et al. [6] descatters a target pattern inside translucent media by combining pattern projection and compressive sensing approach. Our method aims at recovering slices inside translucent objects at arbitrary depths.

Our method falls in a class of active sensing techniques that use high-frequency pattern projection. The original Nayar et al. [26]'s method and its extended methods [18, 21, $22,30,2,1]$ separate direct and global components by projecting multiple high-frequency patterns. Gupta et al. [11] acquire scene depths with direct-global separation by modeling both projector's defocus and global light transport. We also use a pattern projection technique not only for separating scattering effects but also for recovering outer and inner slices.

Methods for measuring transparent or translucent media, such as smoke scanning, are also related to our work. Morris et al. [20] recover the shape of clear transparent materials that refract light by analyzing light rays recorded from different viewpoints. Hawkins et al. [13] acquire the density distribution of participating media by laser scanning, which does not contain multiple scattering, such as smoke. Ihrke and Magnor [15] reconstruct the volume of dynamic semitransparent media by a visual-hull based method. While related, our goal is to recover slices inside translucent objects instead of reconstructing 3-D shape of an object's surface.

In other fields, there have been independent developments of technologies for imaging internal structures of target objects. In art analysis, several techniques have been developed for imaging hidden layers of paintings. Infrared reflectography [9] and X-ray transmission radiography [5] can visualize internal layers of paintings, although the surface texture cannot be separated. X-ray fluorescence technique [5] uses spectroscopic information measured over a couple of days and estimates the metallic atom distribution for determining colored appearance of inner layers. Terahertz imaging [3] is an another technique that can see inner layers of paints. In the medical field and its related areas, optical coherence tomography $[14,7]$ techniques are widely used, especially for visualizing retina. These techniques enable visualizing inside translucent objects using interference of coherent light at the cost of expensive wavelengthorder optics and mechanics. In contrast to these method, our method uses a commodity camera and projector for recovering slices inside translucent objects, resulting in an inexpensive setup. In microscopy, there are methods that use pattern projection for visualizing inside substances, or re-

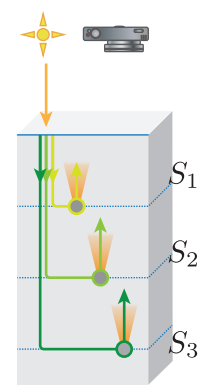

(a)

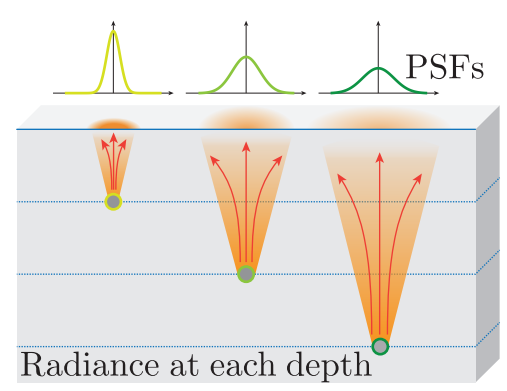

(b)
Figure 1: Appearance of translucent objects. (a) Captured image contains all components from each depth. (b) Lights spread depending on the depth.

constructing 3-D shape of a small sample, such as protein structure $[12,17,31]$. They sharpen the microscopic image by precisely taking into account both micro-scale optical blur and scattering blur. Our aim is to develop a technique that is applicable to a standard scale, where multiple scattering is significant.

\section{Appearance of translucent objects}

When an image of a translucent layered object is recorded, the observed intensity can be modeled as a summation of the appearance of multiple depth layers as illustrated in Fig. 1(a). Let $S_{d}$ be the appearance slice of the layer at depth $d$. The observed intensity $L_{o}(c)$ at camera pixel $c$ can be expressed by

$$
L_{o}(c)=\sum_{d} S_{d}(c)
$$

in a discrete form.

The appearance slice $S_{d}$ is generally blurry due to the scattering effect inside the medium. The spread of radiance at a scene point inside a translucent object varies depending on its depth from the object surface [28]. In general, the spatial spread of light can be expressed using PSFs. Let us consider light rays emitted (or returned) from a specific depth inside a translucent object. When the depth is shallower, the PSF becomes sharper. On the other hand, it gradually spreads as the depth $d$ becomes deeper inside the medium as illustrated in Fig. 1(b). In this manner, there is a close relationship between the PSF and depth. By denoting $h_{d}$ as a PSF at depth $d$, the appearance slice $S_{d}$ at depth $d$ can be expressed as

$$
S_{d}(c)=\left(R_{d} * h_{d}\right)(c),
$$

where $R_{d}$ is the sharp slice that we are interested in estimating, which we call a radiance slice, and $*$ denotes a convolution operator. 
Since the appearance of the translucent object under normal illumination is a superposition of radiance of multiple layers as Eq. (1), the observation $L_{o}$ can be re-written as

$$
L_{o}(c)=\sum_{d}\left(R_{d} * h_{d}\right)(c) .
$$

Our goal is to recover radiance slices $R_{d}$ from the composite observation $L_{o}$. Before introducing the solution method, we describe a model of depth-dependent PSFs $h_{d}$.

Depth-dependent PSFs We represent the depthdependent PSFs $h_{d}$ by a physically motivated scattering model, the radiative transfer equation (RTE) $[4,16]$. With the RTE model, the intensity of an arbitrary light ray in a homogeneous scattering medium can be iteratively calculated [23] as

$$
I(d, \theta)=\sum_{k=0}^{\infty}\left(g_{k}(T)+g_{k+1}(T)\right) L_{k}(\cos \theta),
$$

where $L_{k}$ is the Legendre polynomial of order $k, g_{0}=0$, and

$g_{k}(T)=I_{0} \exp \left(-\frac{2 k+1}{k}\left(1-q^{k-1}\right) T-(k+1) \log T\right)$.

The parameter $T(=\sigma d)$ represents optical thickness, which is the product of extinction coefficient $\sigma$ of scattering media and distance $d$ from the point light source to the scattering point. The forward scattering parameter $q$ controls how light rays spread; $q=0$ corresponds to isotropic, while positive and negative values of $q$ indicate forward and back scattering, respectively. $\theta$ is the angle between the depth axis and light ray's direction as depicted in Fig. 2(a). $k$ is the number of light bounces, and $I_{0}$ is the intensity of the point light source.

In our problem setting, a camera is placed outside the media. When we consider the $x$-axis is on the surface of translucent object as illustrated in Fig. 2(b), the depthdependent PSF $h_{d}(x)$ can be written as

$$
h_{d}(x)=I\left(d^{\prime}, \phi\right),
$$

where $d^{\prime}=\sqrt{x^{2}+d^{2}}$ and $\phi=\tan ^{-1}\left(\frac{x}{d}\right)$. In this work, we assume the camera is placed far enough from the object compared to both $d$ and $x$; therefore, the direction of emitting light rays from the surface becomes parallel to the depth axis. Moreover, we ignore the refraction of the surface for simplicity.

\section{Proposed Method}

We are interested in recovering radiance slices $R_{d}$ from the mixed observation $L_{o}$. To achieve this goal, we develop

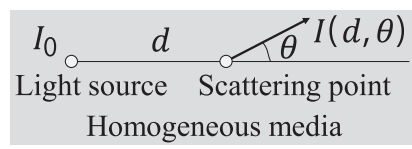

(a)

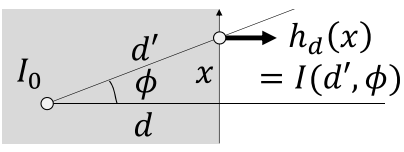

(b)
Figure 2: Illustration of RTE model described in [23]. (a) Black arrow shows an scattering light ray, whose intensity depends on $d$ and $\theta$. $d$ is the distance from the point light source $I_{0}$ inside scattering media and $\theta$ is the radial direction of the light ray. (b) Depth-dependent PSF $h_{d}$ can be expressed using RTE model. We consider the $x$-axis is on the surface of translucent object and assume the direction of emitting light rays from the surface becomes parallel to the depth axis.

a multi-frequency illumination measurement method, which is built upon the high-frequency illumination (HFI) method proposed by Nayar et al. [26]. To begin with, we briefly review the original HFI method.

High-frequency illumination method [26] The HFI method separates direct and global components by projecting small pitch checker patterns. When the phase of the projection pattern changes slightly, the direct component $D(c)$ varies accordingly, but the global component $G(c)$ remains stable. Based on this observation, their method computes direct and global components using the maximum $L_{\max }(c)$ and minimum $L_{\min }(c)$ intensities that are obtained by shifting the projector pattern as

$$
\left\{\begin{array}{l}
D(c)=L_{\max }(c)-L_{\min }(c) \\
G(c)=2 L_{\min }(c)
\end{array}\right.
$$

The direct component $D(c)$ contains high-frequency components, while the global component $G(c)$ contains only lower frequency components than the frequency of projection pattern. Therefore, the HFI method can be viewed as a separation technique for high- and low-frequency components.

Pattern pitch of HFI In our case, when a translucent object is measured under HFI with pattern pitch $p$, we can obtain direct component $D(p, c)$ and global component $G(p, c)$ as

$$
\left\{\begin{array}{rlcc}
D(p, c) & = & \sum_{d} D_{d}(p, c) \\
G(p, c) & = & \sum_{d} G_{d}(p, c) \\
S_{d}(c) & = & D_{d}(p, c)+G_{d}(p, c)
\end{array}\right.
$$

where $D_{d}(p, c)$ and $G_{d}(p, c)$ are the direct (high-frequency) and global (low-frequency) components at depth $d$, respectively. 
When the projected pattern pitch $p$ changes slightly, intensities of direct and global components also change. In fact, there is an important relationship between the pitch size and strength of direct component. Suppose we measure the scene with two variations of pattern pitches $p_{i}$ and $p_{j}\left(p_{i}<p_{j}\right)$ individually. When the pattern pitch becomes larger, wider spread of light rays are included in the direct component, hence the corresponding direct components have the following relationship:

$$
D_{d}\left(p_{i}, c\right)<D_{d}\left(p_{j}, c\right), \quad p_{i}<p_{j} .
$$

This relation indicates that the separation frequency varies with the pattern pitch - wider frequency band is regarded as the direct component as the pattern pitch becomes larger. As low-pass characteristics of depth-dependent PSFs are also depth-dependent, the difference of direct components $D_{d}\left(p_{j}, c\right)-D_{d}\left(p_{i}, c\right)$ also varies with depth $d$. Our method exploits these differences for recovering each inner slice by changing the projection pattern pitch.

\subsection{Multi-frequency illumination}

By measuring the target object using multi-frequency patterns, corresponding multiple direct components are obtained. Unfortunately, increasing the number of measurements does not make the problem easier as it also increases the number of variables to solve for. To make the problem tractable, we assume that the texture of direct components does not vary drastically when the pattern frequency is high enough and the pitch variation $\Delta_{p}\left(=p_{j}-p_{i}\right)$ is sufficiently small. Namely, we assume that direct component images $D_{d}(p, c)$ have linear relationship within a small pitch variations of $p_{i}$ and $p_{j}$ as

$$
D_{d}\left(p_{i}, c\right) \approx \alpha_{\Delta_{p}} D_{d}\left(p_{j}, c\right),
$$

where $\alpha_{\Delta_{p}}$ is the ratio of the mean brightnesses of two individual images. At the same time, these direct components $D_{d}$ at a certain depth $d$ are supposed to have a similar texture with the original radiance; therefore, the following relationship holds:

$$
D_{d}(p, c) \approx \alpha\left(h_{d}, p\right) R_{d}(c),
$$

where $\alpha\left(h_{d}, p\right)$ is a relative brightness of $D_{d}(p, c)$ to $R_{d}(c)$. We call $\alpha\left(h_{d}, p\right)$ the direct component ratio that represents the ratio of direct component's mean intensity to the radiance $R_{d}(c)$ 's mean intensity. Hence, Eq. (7) can be rewritten as

$$
D(p, c)=\sum_{d} \alpha\left(h_{d}, p\right) R_{d}(c) .
$$

With these assumptions, a set of direct component images $D(p, c)$ taken under the multi-frequency illumination of $m$ pitch variations $\left(p=p_{0}, p_{1}, \ldots, p_{m-1}\right)$ can be written in a matrix form as

$$
\mathbf{D}(c)=\mathbf{A R}(c),
$$

where

$$
\begin{aligned}
\mathbf{D}(c) & =\left[\begin{array}{cccc}
D\left(p_{0}, c\right) & D\left(p_{1}, c\right) & \cdots & D\left(p_{m-1}, c\right)
\end{array}\right]^{T}, \\
\mathbf{A} & =\left[\begin{array}{ccc}
\alpha\left(h_{d_{0}}, p_{0}\right) & \cdots & \alpha\left(h_{d_{n-1}}, p_{0}\right) \\
\vdots & \ddots & \vdots \\
\alpha\left(h_{d_{0}}, p_{m-1}\right) & \cdots & \alpha\left(h_{d_{n-1}}, p_{m-1}\right)
\end{array}\right] \\
\mathbf{R}(c) & =\left[\begin{array}{llll}
R_{d_{0}}(c) & R_{d_{1}}(c) & \cdots & R_{d_{n-1}}(c)
\end{array}\right]^{T} .
\end{aligned}
$$

Here, $\mathbf{D}$ is a vector of direct components measured under $m$ variations of the pattern pitches at pixel $c, \mathbf{R}$ is a vector of $n$ layers of radiance slices, and $\mathbf{A}$ is a matrix containing direct component ratios of direct components computed from the projected pattern pitch and the depth-dependent PSF.

When the number of projected patterns $m$ is no less than the number of depth layers $n(m \geq n)$ and $\operatorname{rank}(\mathbf{A})=n$, the radiance slices $\mathbf{R}$ can be obtained in a least-squares sense by computing the pseudo-inverse $\mathbf{A}^{+}$as

$$
\mathbf{R}(c)=\mathbf{A}^{+} \mathbf{D}(c) .
$$

Computation of direct component ratio The direct component ratio $\alpha\left(h_{d}, p\right)$ can be derived from the depthdependent PSF $h_{d}$ and the projected pattern pitch $p$. When checker pattern is projected to a translucent object, it reaches at the depth $d$ with the blur effect, and returns to the surface with the blur effect again. To obtain the direct component ratio, we simply consider the difference of maximum and minimum intensities (as original HFI) under simple conditions: the reflectance of the layer is constant, and illumination is normalized. The direct component ratio can then be obtained as

$$
\begin{aligned}
\alpha\left(h_{d}, p\right)= & \max \left(\left(l_{p} * h_{d}\right) * h_{d}\right) \\
& -\min \left(\left(l_{p} * h_{d}\right) * h_{d}\right),
\end{aligned}
$$

where $l_{p}$ is normalized projection patterns, whose pitch is $p$. Normalized illumination is defined as that 1 to be white and 0 to be black. max and min operators return a maximum and minimum value from all pixels, respectively.

\subsection{Estimation of informative slices}

Once we determine the depths to recover (or PSFs), we can set up a matrix A using Eq. (14), and thus can recover slices corresponding to the PSFs using Eq. (13). Selecting a good set of depths is important for recovering informative slices. For example, if an arbitrary depth is chosen, it may correspond to the middle of distinct texture layers. To recover informative slices, we use a two step approach to 


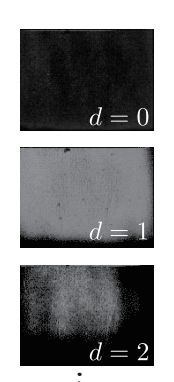

(a)

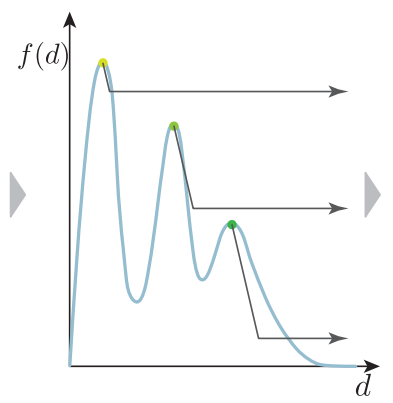

(b)

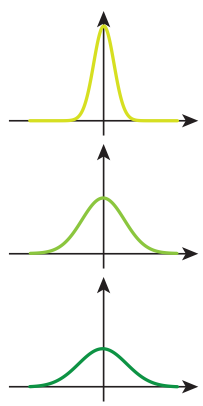

(c)
Figure 3: Selection of informative PSFs (corresponding to depths). We select useful PSFs from a lot of candidate PSFs. (a) Results of the optimization problem (Eq. (15)). Nonzero pixels indicate the informative ones. (b) We find local maxima of the non-zero counts for all pixels across depth. (c) Selected informative PSFs.

determining the matrix A. The first step is the estimation of a set of informative depths via optimization. This is equivalent to estimating the small number of useful PSFs out from many possible PSFs. The second step is the recovery of slices at the informative depths determined by step 1 . The flow of this strategy is illustrated in Fig. 3. Now, we explain the details of each step.

Step 1: Estimation of informative depths Elements of matrix A depend on depth-dependent PSFs; hence estimation of informative depths corresponds to estimating the shape of PSFs. The depth-dependent PSFs depend not on the absolute depth value but on the relative depth $T$, hence we regard it as a depth value. The parameter $T$ of the RTE model are set from 1.01 to 10.01 step by 0.05 . We add the offset 0.01 because Eq. (4) does not converge if $T \leq 1.00$. Initially, we set up the matrix $\mathbf{A}$ using all parameters (thus $m<n)$ in order to estimate informative slices.

Frequently, there are only small number of informative slices inside translucent objects. Hence we can regard such radiance slices exist sparsely along depth. Our method uses this sparsity to determine informative slices by solving a $l_{1}$ regularized problem (as known as the lasso [32]) with a non-negative constraint about $\mathbf{R}$ :

$$
\begin{aligned}
& \tilde{\mathbf{R}}(c)=\underset{\mathbf{R}(c)}{\operatorname{argmin}}\|\mathbf{A R}(c)-\mathbf{D}(c)\|_{2}^{2}+\lambda\|\mathbf{R}(c)\|_{1} \\
& \text { subject to } \mathbf{R} \succeq 0 .
\end{aligned}
$$

It becomes a quadratic programming (QP) problem and thus can be efficiently solved in a polynomial time. We solve the optimization in a per-pixel manner. Solving a similar problem for an entire image is also a viable option; however, we have observed that these two does not make much differences because of the following step 2. Therefore, for efficient parallelization, we choose the per-pixel implementation.

Step 2: Informative slice recovery We can regard the depth $d\left(=\frac{T}{\sigma}\right)$, where $\tilde{R}_{d}(c)$ has a non-zero value, is informative while others are not. This step determines informative depth slices of the whole image by consolidating all the pixel-wise selections. The informative depths $\tilde{\mathbf{d}}$ are local maximas of the sum of $l_{0}$ norm of $\tilde{R}_{d}(c)$ for all pixels. The evaluation function $f(d)$ is defined as

$$
f(d)=\sum_{c}\left\|\tilde{R}_{d}(c)\right\|_{0}
$$

and we find all local maxima of $f(d)$ along $d$ from 0 to 200 as shown in Fig. 3(b). Using the selected depths, we can set up a small sized matrix $\tilde{\mathbf{A}}$. Finally, we can recover informative slices using the matrix $\tilde{\mathbf{A}}$ in a least-square sense as

$$
\mathbf{R}(c)=\tilde{\mathbf{A}}^{+} \mathbf{D}(c)
$$

in the same way as Eq. (13).

\section{Experiments}

We evaluate our method numerically by simulation and also show qualitative results using real-world scenes.

\subsection{Evaluation by simulation}

We first assess the validity of the approximation in Eq. (10), and then evaluate the accuracy of the slice recovery.

Validity of approximation in Eq. (10) In this simulation, we change the pattern pitch $p$ from 3 to 20 pixels, the depth $d$ from 4 to 18 [mm] (in optical depths $T$, which correspond to $4 \sigma$ to $18 \sigma$ ), and use 15 different slice textures. The scene is one-layered for the purpose of assessing the approximation capability. The coefficient $\sigma$ is set to $0.001\left[\mathrm{~mm}^{-1}\right]$, and forward scattering parameter $q$ is set to 0.9 . These parameters are chosen according to [24]. We generate the appearance slices $S_{d, p}^{\varphi}$ using checker pattern $l_{p}^{\varphi}$ as

$$
S_{d, p}^{\varphi}=\left(\left(l_{p}^{\varphi} * h_{d}\right) \circ R_{d}\right) * h_{d} .
$$

$p$ and $\varphi$ are the pitch and phase of the pattern, and $\circ$ is the Hadamard (element-wise) product operator. We compute direct component $D_{d}(p)$ from these synthetic images with changing the phase $\varphi$.

We assess correlations between the recovered direct components $D_{d}(p)$ and the ground truth slices $R_{d}$ using zero-mean cross correlation (ZNCC). The ZNCC value falls 


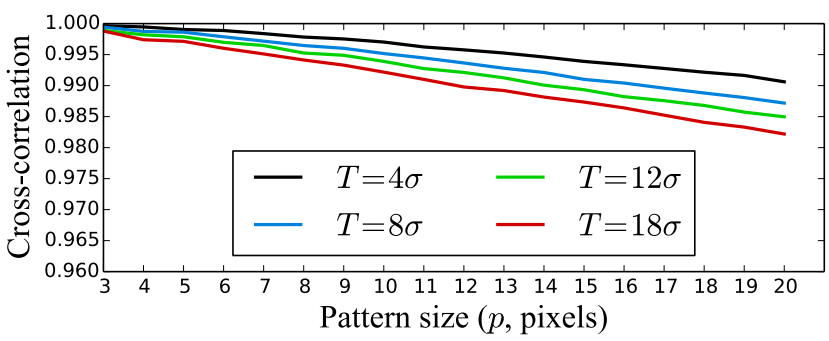

Figure 4: Evaluation result of the approximation in Eq. (10). We calculate the cross correlation between direct component slice $D_{d}(p)$ and the ground truth slice $R_{d}$. Correlation values are very high even with the worst case.

in the range from -1 to 1 , where a negative value indicates negative correlation, and a greater value indicates higher similarity. The evaluation results are summarized in Fig. 4. The plots indicate mean correlations of all slice textures. ZNCC values decreases as the depth becomes deeper (greater $T$ ) and pattern pitch becomes larger. However, they are all consistently well correlated (minimum $\mathrm{ZNCC}$ value is 0.982 and the mean value is 0.992 ), and it shows the accuracy of the approximation.

Evaluation of slice recovery We also evaluate how accurately slices can be recovered by our method via simulation. We generate images $L_{p}^{\varphi}$ under projection patterns $l_{p}^{\varphi}$ as

$$
L_{p}^{\varphi}=\sum_{d}\left(\left(l_{p}^{\varphi} * h_{d}\right) \circ R_{d}\right) * h_{d} .
$$

We use 20 different two-layered scenes that have distinct textures $R_{5}$ and $R_{15}$ at two depth ranges $5 \leq d<15$ and $15 \leq d$. The textures are randomly paired from the ones that are used in the previous experiment. We change the pitch $p$ of checker pattern and shift the pattern with $\varphi$. From the generated images, we compute $D(p, c)$ for each pattern pitch $p$, and apply our method to recover slices at $d=5$ and 15.

We again use ZNCC values between the recovered and ground truth slices. The experimental results are shown in Table 1. In all sets, the ZNCC values of upper layer is higher than the lower layer as expected. The result of this simulation indicates that our method can recover slices of various textures with high correlation.

Figure 5 shows a synthetic example of three-layer recovery, where slices at at $d=1,5$, and 15 are recovered. The ZNCC scores for the recovery results are $0.98,0.83$, and 0.51 , respectively. While the result is generally consistent with the ground truth, negative intensities and ringing artifacts are observed due to the discrete pitch variations and convolution.

\begin{tabular}{|c|ccc|}
\hline & \multicolumn{3}{|c|}{ ZNCC values } \\
Layers & Max. & Mean & Min. \\
\hline \hline Top layer & 0.99 & 0.93 & 0.89 \\
Bottom layer & 0.92 & 0.84 & 0.67 \\
\hline
\end{tabular}

Table 1: 2-layer recovery result for 20 sets of scenes. We compare recovered slices to the ground truth slices by ZNCC. Higher ZNCC scores indicate more accurate recovery.

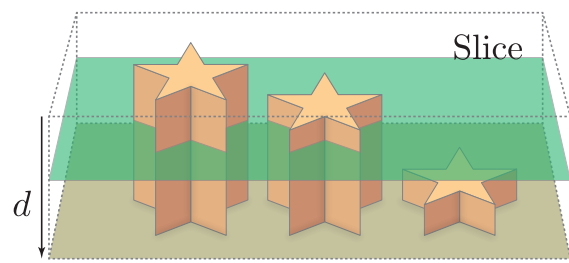

(a) Simulated scene. three star-shaped pillars with different heights are placed in scattering media. An example slice is illustrated by the orange plane.

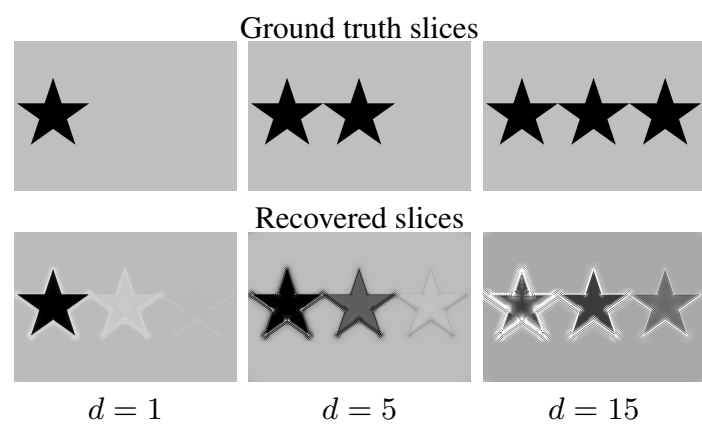

(b) Comparison of the recovered and ground truth slices

Figure 5: (a) Target scene (b) Result of three-layer recovery at $d=1,5$, and 15 . The ground truth radiances slices (upper) and recovered slices (lower) are shown. ZNCC scores are $0.98,0.83$, and 0.51 , respectively.

\subsection{Real-world experiment}

We develop a coaxial projector-camera setup for realizing the measurement setup as shown in Fig. 6. The coaxial setup has a favorable property, i.e., correspondence between projector and camera pixels becomes invariant with respect to depths. Unlike non-coaxial settings, with which a illumination ray inside the translucent object forms a line in the image coordinates [30], the coaxial setting allows us to easily separate the direct rays. We use a "LightCommander" projector, which is a DMD projector development kit by Texas Instruments, and use near infrared (NIR) light for measurements. The lenses of both camera and projector are set same (Ai Micro-Nikkor $105 \mathrm{~mm} \mathrm{f} / 2.8 \mathrm{~S}$ ) for easy alignment. In the experiment, we use 18 variations of checker patterns (3px to 20px with 1px interval), and shift the pattern for one-third of square size for each pattern. 


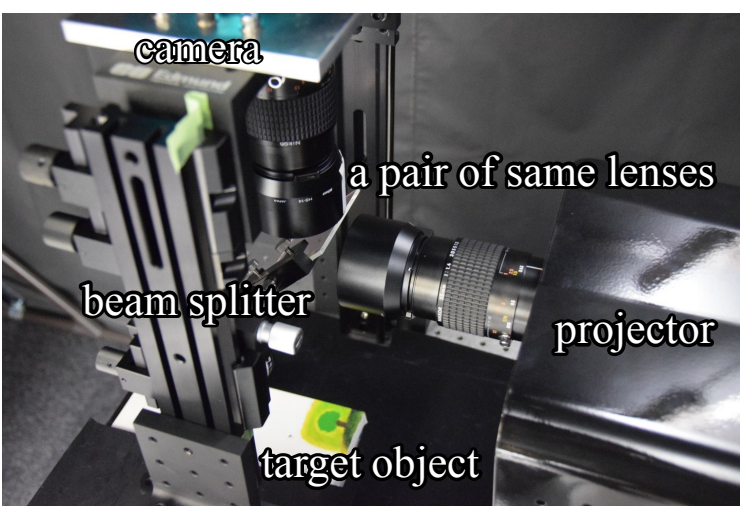

Figure 6: Measurement setup. The coaxial system allows us to maintain the correspondences between projector and camera pixels.

Experimental results First, we use an oil painting as a target scene as shown in Fig. 7(a), which has draft and surface pigment layers as depicted in Figs. 7(b) and 7(c). By taking a standard photograph under the near infrared light (as done in infrared reflectography in the art field), we can vaguely observe the draft layer as shown in Fig. 7(d). This is, however, the superposition of draft and surface pigment layers, hence it results in a blurry image. Therefore, a simple layer decomposition still suffers from the blur. We cannot see the shape of the draft tree clearly even if we manually adjust the contrast, which is essentially same as infrared reflectography [9], as shown in Fig. 7(e). By applying our method to this scene, two PSFs are estimated as depicted in Fig. 7(h) and two slices are recovered as in Figs. 7(f) and $7(\mathrm{~g})$. The upper surface layer corresponds to the surface pigment layer. Because the yellow pigment is almost transparent in the infrared wavelength, the corresponding painting regions become dark in the surface slice. The lower layer shows the inner layer, where the texture of the tree is clearly observed.

The second target scene is stacked papers that consist of layered translucent papers and printed texture films as shown in Fig. 8(a). In a normal NIR photograph, we can observe mixed textures as shown in Fig. 8(b), where textures are blurry and appearances across depths are superpositioned. Our method correctly selects two informative depths and recovers radiance slices as shown in Figs. 8(c) and $8(\mathrm{~d})$. In the upper slice, only 'ABCD' texture is visible, and '1234' texture appears in the lower slice. Due to the shadowing effect caused by the solid material on the upper layer (the texture of 'ABCD'), the lower slice is contains the 'ABCD' texture as an unobserved shadowed region.

Additional results are shown in Fig. 9. The upper row is a piece of painting, where the painter's signature is hidden under pigment. Our method clearly recovers surface texture and inner signature slices. The lower row is a mural painting covered by white mold. In this example, we used RGB

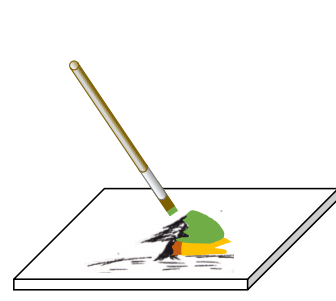

(a) The scene

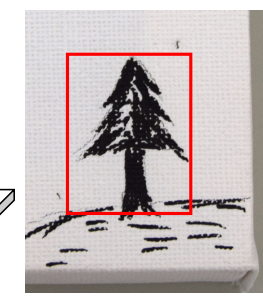

(b) Draft layer

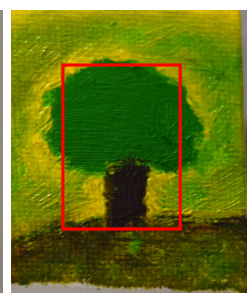

(c) Oil painted

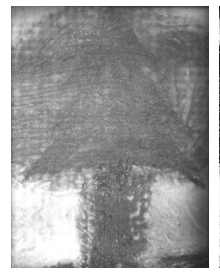

(d) NIR photo

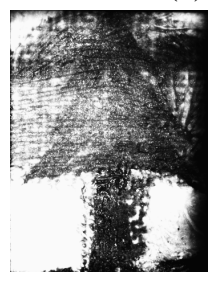

(e) Baseline

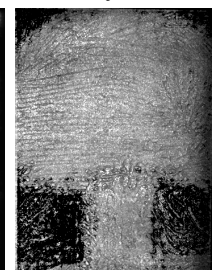

(f) Ours

(surface slice)

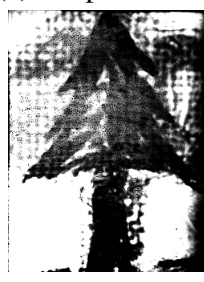

(g) Ours (inner slice)

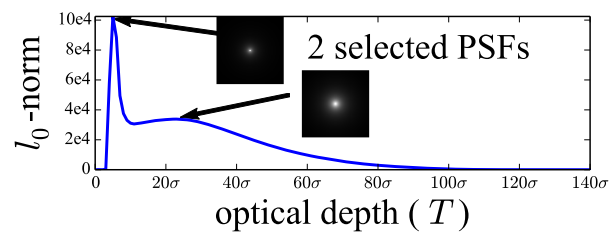

(h) Selected PSFs corresponding to local maxima

Figure 7: Experimental result of oil painting. (a) Target scene. We draw a colored round tree on top of the draft of spiny tree. (b) Inner layer (draft) of the painting. (c) Painted scene. Red rectangle region is measured. (d) Normal photo using infrared light. (e) Baseline result. Intensity range and contrast of (d) are manually adjusted. (f, g) Results of our method. Layer of surface texture and hidden drawing, respectively. Range of the intensities is adjusted for visualization. (h) Selected PSFs. There are two peaks in the plot of Eq. (16), hence two corresponding PSFs are selected to recover.

light sources instead of NIR, and record each color channel separately. Our method can recover the slices in this example as well, and the inner appearance is clearly visible in the result of Fig. 9(d).

Thickness estimation Our method can be applied to estimate the thickness of pigments by computing Eq. (16) in a patch-based manner instead of the entire image region. As we do not know the scattering coefficient, the estimated thickness is relative (but linear). Figure 10(a) is a scene, where brown pigment is drawn on a white paper. We find the relative depth of a pixel using a $9 \times 9$ image patch centered at the pixel. The estimated thickness is shown in Fig. 10(b), where the estimated relative thickness is shown in a pseudo color map. 

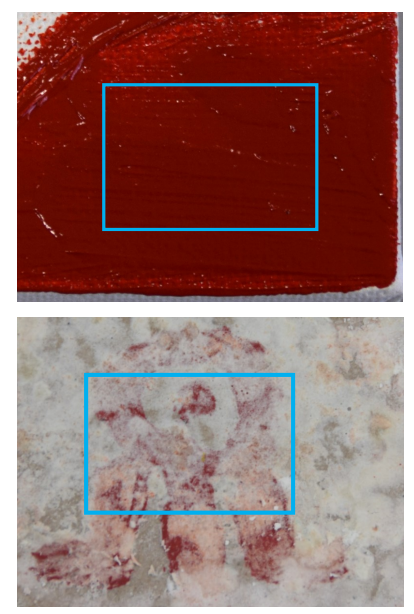

(a) Target scene
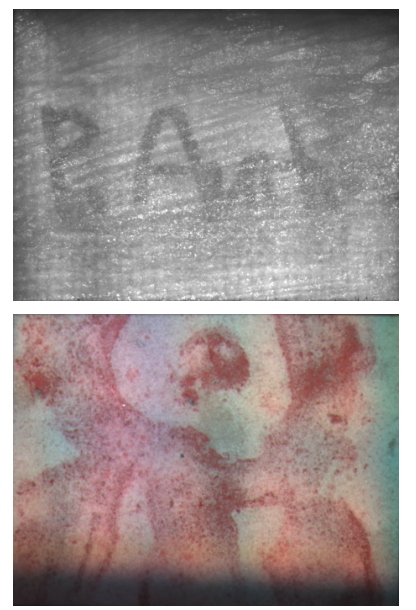

(b) Normal photo
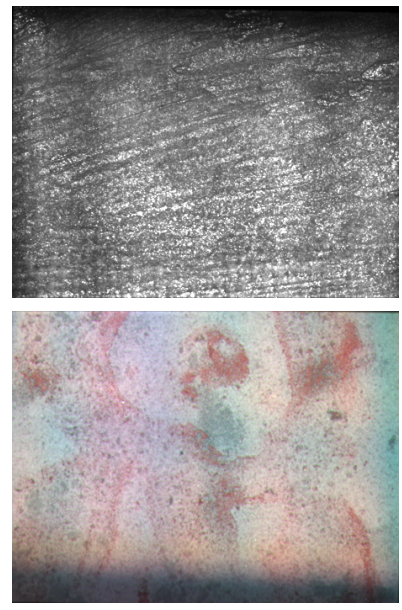

(c) Surface slice
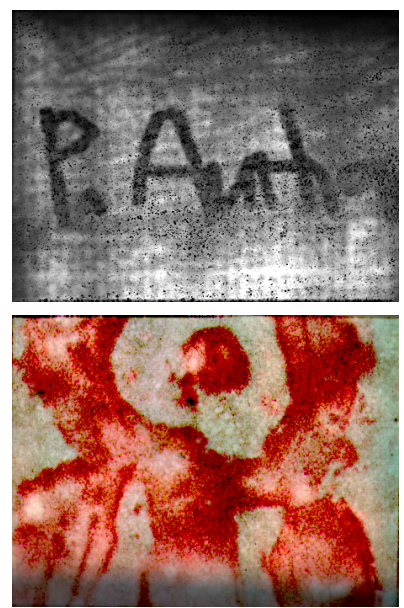

(d) Inner slice

Figure 9: Additional results for a painting and a mural. (a) Target scenes. Upper: author's signature covered by red pigment. Lower: A mural painting covered by white mold. Rectangle regions are measured. (b) Normal photograph. Both upper and lower slices, and global components are composed. (c) Recovered slices of surface. (d) Recovered inner slices. The signature and inner painting are visible clearly.

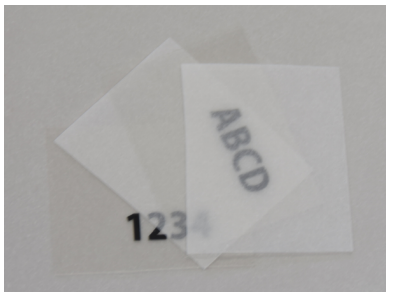

(a) The scene

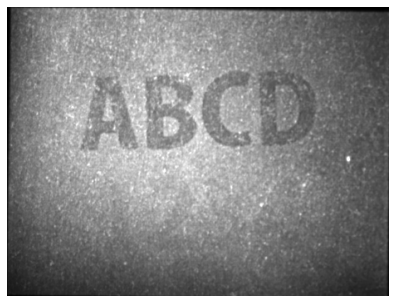

(c) Upper slice

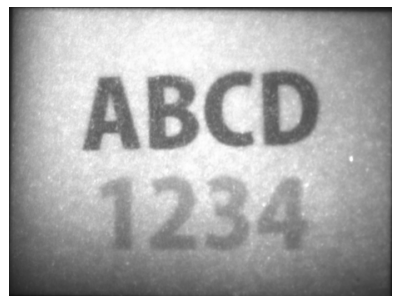

(b) NIR photo

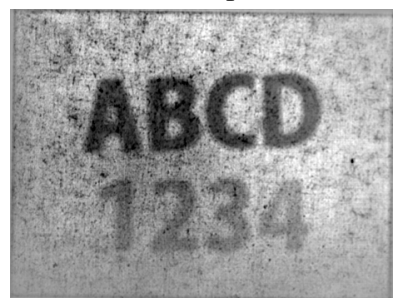

(d) Lower slice
Figure 8: Experimental result of layered scene. (a) Scene is a composite object of texture and translucent sheets (tracing paper). (b) Normal photo of the scene. Textures inside the object can be seen, although blurry. (c) Recovered slice of upper layer. (d) Recovered slice of lower layer. Textures in sub-millimeter gap can be separated.

\section{Discussions}

This paper described a method for recovering inner slices of translucent objects based on multi-frequency pattern projection. We have shown that inner radiance slices can be obtained by varying pitches of projection patterns. We also developed a method for automatically selecting informative slices via a sparse representation, i.e., determining sparse coefficients for recovery of the observations. The effective-

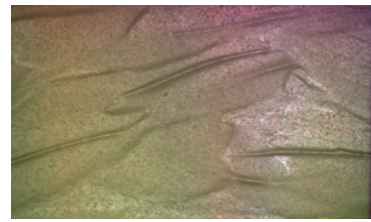

(a) Non-smooth painting

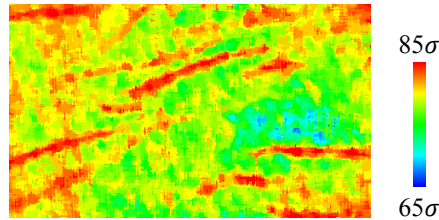

(b) Relative thickness
Figure 10: Pigment thickness estimation. By selecting depth-dependent PSFs in a patch-based manner, our method can estimate the relative thickness of the pigment. $\sigma$ is an unknown scattering parameter, which works as scale factor.

ness of the proposed method is shown by several experiments of simulation and real-world translucent objects.

There are future works that we are interested in exploring. First, the result is affected by an opaque object in upper layers due to that the lower layers are shadowed. In other words, the lower layer's reflectance is regarded to be zero, and as a result, we observe zero-reflectance textures in the lower layers. While this issue is difficult to directly address in the proposed scheme, we are interested in resolving this issue, or at least identify the shadowed regions, by assessing the correlation between upper and lower textures and their reflectance. Second, because our method requires multiple images under varying pitches, the measurement takes approximately ten minutes in our experiments. We are interested in developing a more efficient measurement technique by exploiting multiplexing in space and wavelength domains.

\section{References}

[1] S. Achar and S. G. Narasimhan. Multi Focus Structured Light for Recovering Scene Shape and Global Illumination. 
In European Conference on Computer Vision (ECCV), 2014. 2

[2] S. Achar, S. T. Nuske, and S. G. Narasimhan. Compensating for Motion during Direct-Global Separation. In IEEE International Conference on Computer Vision (ICCV), 2013. 2

[3] A. J. L. Adam, P. C. M. Planken, S. Meloni, and J. Dik. TeraHertz imaging of hidden paint layers on canvas. Optics Express, 17(5):3407, Feb. 2009. 2

[4] S. Chandrasekhar. Radiative Transfer. Dover Publications, Inc., 1960. 3

[5] J. Dik, K. Janssens, G. Van der Snickt, L. van der Loeff, K. Rickers, and M. Cotte. Visualization of a lost painting by Vincent van Gogh using synchrotron radiation based Xray fluorescence elemental mapping. Analytical chemistry, 80(16):6436-42, Aug. 2008. 1, 2

[6] V. Durán, F. Soldevila, E. Irles, P. Clemente, E. Tajahuerce, P. Andrés, and J. Lancis. Imaging at depth in tissue with a single-pixel camera. arXiv, Nov. 2014. 2

[7] A. F. Fercher. Optical coherence tomography. Journal of Biomedical Optics, 1(2):157-173, 1996. 2

[8] C. Fuchs, M. Heinz, M. Levoy, H.-P. Seidel, and H. P. A. Lensch. Combining confocal imaging and descattering. Computer Graphics Forum, Special Issue for the Eurographics Symposium on Rendering 2008, 27(4):1245-1253, 6 2008. 2

[9] D. Gavrilov, E. Maeva, O. Grube, X. Maldague, and R. Maev. Infrared Methods in Noninvasive Inspection of Artwork. In International Conference on NDT of Art, number May, pages 1-5, 2008. 1, 2, 7

[10] J. Gu, S. K. Nayar, E. Grinspun, P. Belhumeur, and R. Ramamoorthi. Compressive Structured Light for Recovering Inhomogeneous Participating Media. IEEE Transactions on Pattern Analysis and Machine Intelligence(TPAMI), 35(3):555-567, 2013. 2

[11] M. Gupta, Y. Tian, S. G. Narasimhan, and L. Zhang. A Combined Theory of Defocused Illumination and Global Light Transport. International Journal of Computer Vision, 98(2):146-167, Oct. 2011. 2

[12] M. G. L. Gustafsson, L. Shao, P. M. Carlton, C. J. R. Wang, I. N. Golubovskaya, W. Z. Cande, D. A. Agard, and J. W. Sedat. Three-dimensional resolution doubling in wide-field fluorescence microscopy by structured illumination. Biophysical journal, 94(12):4957-70, June 2008. 2

[13] T. Hawkins, P. Einarsson, and P. Debevec. Acquisition of time-varying participating media. ACM Transaction on Graphics, 24(3):812-815, July 2005. 2

[14] D. Huang, E. Swanson, C. Lin, J. Schuman, W. Stinson, W. Chang, M. Hee, T. Flotte, K. Gregory, C. Puliafito, and A. Et. Optical coherence tomography. Science, 254(5035):1178-1181, Nov. 1991. 2

[15] I. Ihrke and M. Magnor. Adaptive grid optical tomography. Graphical Models, 68(5):484 - 495, 2006. Special Issue on the Vision, Video and Graphics Conference 2005. 2

[16] A. Ishimaru. Wave Propagation and Scattering in Random Media. IEEE Press, 1997. 3

[17] P. Kner, B. B. Chhun, E. R. Griffis, L. Winoto, and M. G. L. Gustafsson. Super-resolution video microscopy of live cells by structured illumination. Nature methods, 6(5):339-42, May 2009. 2

[18] B. Lamond, P. Peers, and P. Debevec. Fast image-based separation of diffuse and specular reflections. In ACM SIGGRAPH sketches, 2007. 2

[19] Y. Li and M. S. Brown. Single Image Layer Separation using Relative Smoothness. In IEEE Conference on Computer Vision and Pattern Recognition (CVPR), 2014. 1

[20] N. J. W. Morris and K. N. Kutulakos. Reconstructing the Surface of Inhomogeneous Transparent Scenes by Scatter Trace Photography. In International Conference on Computer Vision (ICCV), 2007. 2

[21] Y. Mukaigawa, R. Raskar, and Y. Yagi. Analysis of light transport in scattering media. In IEEE Conference on Computer Vision and Pattern Recognition (CVPR), pages 153160, 2010. 2

[22] Y. Mukaigawa, R. Raskar, and Y. Yagi. Analysis of scattering light transport in translucent media. IPSJ Transactions on Computer Vision and Applications, 3:122-133, 2011. 2

[23] S. Narasimhan and S. Nayar. Shedding light on the weather. In Proceedings of the IEEE Conference on Computer Vision and Pattern Recognition (CVPR), pages 665-672, 2003. 3

[24] S. G. Narasimhan, M. Gupta, C. Donner, R. Ramamoorthi, S. K. Nayar, and H. W. Jensen. Acquiring scattering properties of participating media by dilution. ACM Transactions on Graphics, 25, 2006. 5

[25] S. G. Narasimhan, S. K. Nayar, B. Sun, and S. J. Koppal. Structured light in scattering media. In IEEE International Conference on Computer Vision (ICCV), pages 420427, 2005. 2

[26] S. K. Nayar, G. Krishnan, M. D. Grossberg, and R. Raskar. Fast separation of direct and global components of a scene using high frequency illumination. In Proceeding ACM SIGGRAPH, pages 935-944, 2006. 1, 2, 3

[27] B. Sarel and M. Irani. Separating transparent layers through layer information exchange. In European Conference on Computer Vision (ECCV), 2004. 1

[28] K. Shimizu, K. Tochio, and Y. Kato. Improvement of transcutaneous fluorescent images with a depth-dependent pointspread function. Applied Optics, 44(11):2154-2161, 2005. 2

[29] R. Szeliski, S. Avidan, and P. Anandan. Layer extraction from multiple images containing reflections and transparency. In IEEE Conference on Computer Vision and Pattern Recognition (CVPR), pages 246-253, 2000. 1

[30] K. Tanaka, Y. Mukaigawa, Y. Matsushita, and Y. Yagi. Descattering of transmissive observation using Parallel High-Frequency Illumination. In IEEE International Conference on Computational Photography (ICCP), 2013. 2, 6

[31] O. D. Therrien, B. Aubé, S. Pagès, P. D. Koninck, and D. Côté. Wide-field multiphoton imaging of cellular dynamics in thick tissue by temporal focusing and patterned illumination. Biomedical optics express, 2(3):696-704, 2011. 2

[32] R. Tibshirani. Regression shrinkage and selection via the lasso. Journal of the Royal Statistical Society, Series B, 58:267-288, 1994. 5 